Article

Subscriber access provided by Brought to you by ST ANDREWS UNIVERSITY LIBRARY

\title{
Erythritol Attenuates postprandial blood glucose by inhibiting \#-glucosidase
}

huaixiu wen, bowen tang, Alan J. Stewart, yulei cui, huilan yue, jinjin pei, zenggen liu, yun shao, lijuan mei, yanduo tao, ruitao yu, Lei Jiang, and Lei Jiang

J. Agric. Food Chem., Just Accepted Manuscript • DOI: 10.1021/acs.jafc.7b05033 • Publication Date (Web): 23 Jan 2018

Downloaded from http://pubs.acs.org on February 5, 2018

\section{Just Accepted}

"Just Accepted" manuscripts have been peer-reviewed and accepted for publication. They are posted online prior to technical editing, formatting for publication and author proofing. The American Chemical Society provides "Just Accepted" as a service to the research community to expedite the dissemination of scientific material as soon as possible after acceptance. "Just Accepted" manuscripts appear in full in PDF format accompanied by an HTML abstract. "Just Accepted" manuscripts have been fully peer reviewed, but should not be considered the official version of record. They are citable by the Digital Object Identifier (DOI®). "Just Accepted" is an optional service offered to authors. Therefore, the "Just Accepted" Web site may not include all articles that will be published in the journal. After a manuscript is technically edited and formatted, it will be removed from the "Just Accepted" Web site and published as an ASAP article. Note that technical editing may introduce minor changes to the manuscript text and/or graphics which could affect content, and all legal disclaimers and ethical guidelines that apply to the journal pertain. ACS cannot be held responsible for errors or consequences arising from the use of information contained in these "Just Accepted" manuscripts. 


\section{$1 \quad$ Erythritol attenuates postprandial blood glucose by inhibiting $\alpha$-glucosidase}

2 Huaixiu Wen†, Bowen Tangt, Alan J. Stewart§, Yanduo Tao $\dagger$, Yun Shao $\dagger$, Yulei Cui†,

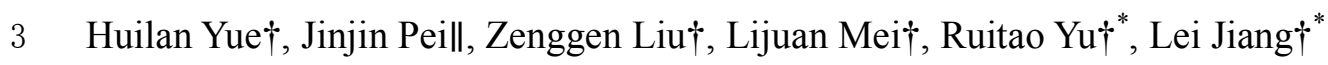

$4 \uparrow$ Key Laboratory of Tibetan Medicine Research, Northwest Plateau Institute of

5 Biology, Chinese Academy of Sciences, Xining 810001, PR China

$6 \$$ School of Pharmaceutical Sciences, Xiamen University, Xiamen 361005, PR China

7 §School of Medicine, University of St Andrews, St Andrews, KY16 9TF, United

8 Kingdom.

9 IIShaanxi Key Laboratory of Bioresources and Biology, Shaanxi University of

10 Technology, Hanzhong 723001, PR China

11 Correspondence: Lei Jiang

12 No. 23 Xinning Road, Xining, Qinghai, China

$13 \quad$ Tel +8615297217193

14 jianglei@nwipb.cas.cn

15 The animal ethical approval number is NWIPB-2016-33.

16 Additional corresponding author: Ruitao $\mathrm{Yu}$

17 No. 23 Xinning Road, Xining, Qinghai, China

18 yuruitao@nwipb.cas.cn

19 Huaixiu Wen and Bowen Tang contributed equally to this work. 


\section{Abstract}

Diabetes mellitus (DM) is a serious metabolic disorder where impaired postprandial blood glucose regulation often leads to severe health complications. The natural chemical, erythritol is a C4 polyol approved by FDA for use as a sweetener. Here we examined a potential role for erythritol in the control of postprandial blood glucose levels in DM. An anti-postprandial hyperglycemia effect upon erythritol administration (500 $\mathrm{mg} \mathrm{kg}^{-1}$ ) was demonstrated in alloxan-induced DM model mice by monitoring changes in blood glucose after intragastric administration of drugs and starch. We also found that erythritol most likely exerts its anti-postprandial hyperglycemic activities by inhibiting $\alpha$-glucosidase in a competitive manner. This was supported by enzyme activity assays and molecular modelling experiments. In the latter experiments it was possible to successful dock erythritol into the catalytic pocket of $\alpha$-glucosidase, with the resultant interaction likely to be driven by electrostatic interactions involving Asp 215, Asp69 and Arg446 residues. This study suggests that erythritol may not only serve as a glucose substitute but may also be a useful agent in the treatment of diabetes mellitus to help manage postprandial blood glucose levels.:

\section{.}

Key words: Diabetes mellitus; postprandial blood glucose; erythritol; $\alpha$-glucosidase; 
competitive inhibition.

\section{Introduction}

Diabetes mellitus (DM), is a disorder characterized by high blood glucose levels $(>126 \mathrm{mg} / \mathrm{dL})$ that affects 415 million people worldwide. DM is recognized as a serious public health concern with the total number of those affected estimated to increase to 642 million by $2040^{1}$. DM is associated with serious health complications including kidney failure, cardiovascular disease, visual impairment, cognitive decline and premature death ${ }^{2,3-4}$. Therefore, new effective therapies for DM are urgently required.

Type $2 \mathrm{DM}$ is identified as a staged reduction of insulin secretion in response to food glucose intake; hence, its primary pathological phenomenon is impaired postprandial glucose regulation ${ }^{5}$. However, clinicians still depend on fasting blood glucose and glycated hemoglobin to guide treatment ${ }^{6}$. It is reported that a linear relationship between the risk of death from cardiovascular disease and the oral glucose tolerance is observed ${ }^{7-8}$. Meanwhile, another study confirms postprandial hyperglycemia to be a major risk factor for cardiovascular disease in individuals with type $2 \mathrm{DM}^{9-10}$. It is reported that postprandial hyperglycemia may promote cardiovascular disease by increasing oxidative stress ${ }^{11}$. Furthermore, it is suggested that postprandial hyperglycemia is a common phenomenon for individuals without $\mathrm{DM}^{12}$. Adequate control of postprandial glucose levels is thus very important and 
66 should be given more attention ${ }^{13}$.

67 In type $2 \mathrm{DM}, \alpha$-glucosidase inhibitors are commonly used to control 83 added to the diets of DM patients ${ }^{17}$. for $\mathrm{DM}^{15}$. postprandial hyperglycemia ${ }^{14}$. The enzyme $\alpha$-Glucosidase is a carbohydrase located on the surface intestinal epithelial cells, where it catalyses the hydrolysis of oligosaccharides into monosaccharides (such as glucose) to facilitate absorption. By significantly delaying intestinal glucose absorption, $\alpha$-glucosidase inhibitors can reduce postprandial hyperglycemia to lower the risk of late diabetic complications ${ }^{14}$. Because of its tolerability and cardiovascular benefits, the International Diabetes Federation (IDF) have recommended $\alpha$-glucosidase inhibitors as a first line therapy

Many sugar derivatives such as sugar alcohols, due to their low-energy character, have been developed into food additives as substitutes for glucose ${ }^{16}$. The sugar alcohol, erythritol exists naturally in algae, wine, sake, beer, pears, grapes, watermelons, and mushrooms and is a C4 polyol, which was approved in 1997 by the FDA to be listed in GRAS (Generally Recognized As Safe List). More than 90\% of ingested erythritol is not metabolized by the human body and is excreted unchanged in the urine. Consequently, erythritol has a potential use as a sweetener that may be

In a previous study, the effects of erythritol on blood glucose in streptozotocin (STZ)-induced DM rats was assessed. It was found that erythritol had a long-term blood glucose controlling capacity, that led to reduced kidney damage caused by $\mathrm{DM}^{18}$. Here we demonstrate that that erythritol is effective for controlling 
postprandial hyperglycemia in alloxan-induced DM model mice and show that it

89 likely mediates this effect by acting as a competitive inhibitor of $\alpha$-glucosidase.

\section{$91 \quad$ Materials and methods}

\section{Chemicals and reagents}

For the outlined studies, $\alpha$-Glucosidase from Saccharomyces cerevisiae (EC 3.2.1.20), erythritol, p-nitrophenyl- $\alpha$-D-glucopyranoside (pNPG) and acarbose were obtained from Sigma-Aldrich Chemical Co. (St. Louis, MO, USA). All other chemicals used were of analytical grade.

\section{Animals}

Thirty SPF grade male Swiss albino male mice (balb/c strain), eight weeks old, were obtained from the SLAC Company Limited (Shanghai, China). Diabetes was induced by a single intravenous injection of aqueous alloxan monohydrate (200 $\mathrm{mg} / \mathrm{kg})$. After $48 \mathrm{~h}$, animals with the serum glucose level within the range (17-24 $\mathrm{mmol} / \mathrm{L}$ ) were selected for the further study. All the animals were housed 5 per cage at $25{ }^{\circ} \mathrm{C}$ with a $12 \mathrm{~h}$ light $/ 12 \mathrm{~h}$ dark cycle. All experiments were approved by the Animal Ethics Committee of Chinese Academy of Sciences and were performed according to Guidelines for the Care and Use of Laboratory Animals. The animal ethical approval number is NWIPB-2016-33.

The thirty mice were randomly allocated into six groups: sham operation plus water group (Sham $\mathrm{Cn}$ ), sham plus acarbose solution (acarbose $4 \mathrm{mg} / \mathrm{Kg} / \mathrm{d}$ ), sham 
110

111

plus erythritol solution (erythritol $500 \mathrm{mg} / \mathrm{Kg} / \mathrm{d}$ ), DM plus water (DM Cn), DM plus acarbose solution group (acarbose $4 \mathrm{mg} / \mathrm{Kg} / \mathrm{d}$ ) and DM plus erythritol solution (erythritol $500 \mathrm{mg} / \mathrm{Kg} / \mathrm{d}$ ). Erythritol was dissolved in water (vehicle) and was administered by intragastric administration with $0.5 \mathrm{~g}$ starch after fasting for 12 hours. After intragastric administration, the blood glucose levels were measured at $0,30,60$, 100,140 and $180 \mathrm{~min}$.

\section{Assessment of $\alpha$-Glucosidase activity}

The $\alpha$-glucosidase activity assay was performed according to a slightly modified method of that previously reported ${ }^{19}$. The $\alpha$-glucosidases $(0.35 \mathrm{U} / \mathrm{mL})$ and substrate (1.5 mM p-nitrophenyl- $\alpha$-D-glucopyranoside) were dissolved in $0.1 \mathrm{M}$ sodium phosphate buffer, $\mathrm{pH}$ 7.0. For this $50 \mu \mathrm{L}$ of acarbose or erythritol was pre-incubated with $100 \mu \mathrm{L}$ of $\alpha$-glucosidase at $37^{\circ} \mathrm{C}$ for $10 \mathrm{~min}$. The substrate solution $(100 \mu \mathrm{L})$ was then added to the reaction mixture and incubated at $37^{\circ} \mathrm{C}$ for $20 \mathrm{~min}$. The reaction was then terminated by adding $1 \mathrm{ml}$ of $1 \mathrm{M} \mathrm{Na}_{2} \mathrm{CO}_{3}$. The absorbance at $405 \mathrm{~nm}$ was determined using a microplate Reader (EnVision; PerkinElmer, USA). All samples were analyzed in triplicate and the percentage of activity was calculated as:

$$
\text { Percentage activity }(\%)=\frac{A_{405}(\text { sample })}{A_{405}(\text { control })} \times 100 \%
$$

Kinetic studies were also performed based on the $\alpha$-glucosidase assay described above. The concentration of $\alpha$-glucosidase was kept constant at $0.1 \mathrm{unit} / \mathrm{mL}$ and the pNPG concentrations varied from 0.11 to $0.51 \mathrm{mM}$ in the absence and presence of 
131

132

133

134

135

erythritol $(5,10$ and $15 \mathrm{mg} / \mathrm{mL})$. The type of inhibition was determined using $\mathrm{V}_{\max }$ and $\mathrm{K}_{\mathrm{m}}$ values obtained using a Lineweaver-Burke plot obtained by plotting velocities of reaction (vertical axis) and substrate concentrations (horizontal axis) reciprocally.

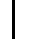

\section{Molecular modeling}

Molecular docking studies were performed using Discovery studio v2.5 software (Accelrys Inc, San Diego, USA). The 3D structure of the $\alpha$-glucosidase, isomaltase from Saccharomyces cerevisiae (PDB: 3AJ7) was obtained from Protein Data Bank (www.rcsb.org). This structure was chosen based on its very high resolution (1.3 $\AA$ ). Crystallographic disorders and unfilled valence atoms were corrected by alternate conformations and valence monitor tools. The molecular docking of erythritol was performed using the Libdock method. To begin the docking, hydrogen bonds were added firstly, and then the energy was minimized by CHARMM force field. After the above steps of preparations, the active site of the enzyme was identified as the binding site to start the docking protocol.

The AMBER 11.0 software was employed to run all the molecular dynamics studies. Erythritol was minimized by the Discovery studio v2.5 software. General Amber Force Field (GAFF) parameters were firstly assigned to the ligands, while partial charges were calculated using the AM1-BCC method of AMBER 11.0. The erythritol-enzyme complex was charge neutralized by adding 10 sodium counter_ions, and then were surrounded by a periodic box of TIP3P water molecules extending up to $10 \AA$ from the solute. First, energy minimizations using a steepest descent method, 
153

followed by the conjugate gradient method, were performed for each system. Then, each system was gradually heated from $0 \mathrm{~K}$ to $300 \mathrm{~K}$ within $30 \mathrm{ps}$. This was followed by a further 500 ps of equilibration at $300 \mathrm{~K}$ carried out to obtain a stable density. Afterward, an unconstrained production phase was initiated and continued for $40 \mathrm{~ns}$ in an NPT ensemble at $1 \mathrm{~atm}$ and $300 \mathrm{~K}$. During the simulations, the long-range electrostatic interactions were evaluated by the Particle Mesh Ewald (PME) algorithm. The cutoff distance for the long-range van der Waals interaction was set to $8 \AA$. The SHAKE method was applied to con-strain the bond lengths of hydrogen atoms attached to heteroatoms. The time step used for the MD simulations was set to $2.0 \mathrm{fs}$ and the trajectory files were collected every 1 ps for the subsequent analysis.

The interaction between inhibitor and each residue was computed using the MM/GBSA decomposition process by the mm_pbsa program in AMBER 11.0. The binding interaction of each inhibitor-residue pair includes three energy terms: van der Waals contribution (vdw), electrostatic contribution (ele) and polar solvation contribution (Polar E) and non-polar solvation contribution (non-Polar E). All energy components were calculated using the 300 snapshots extracted from the MD trajectory from 1.0 to $4.0 \mathrm{~ns}$.

\section{Results and discussion}

\section{Reduction of postprandial blood glucose by erythritol}

The postprandial hypoglycemic effect of erythritol in an alloxan-induced DM mouse model was analyzed. Blood glucose was measured before and 30, 60, 100 and 
140 min after intragastric administration of starch. The blood glucose increased and peaked at 30 minutes in all the groups. Compared with the sham operation plus water group (Sham $\mathrm{Cn}$ ), the sham plus acarbose solution (acarbose $4 \mathrm{mg} / \mathrm{Kg} / \mathrm{d}$ ) and sham plus erythritol solution (erythritol $500 \mathrm{mg} / \mathrm{Kg} / \mathrm{d}$ ) groups both displayed a decrease in average blood glucose but the differences between the three groups were not considered to be significant (Figure 1A). Meanwhile, the area under the concentration-time curve (AUC) of the above three groups did not differ significantly (Figure 1B). The results indicated that erythritol and acarbose did not induce any significant postprandial blood glucose changes in healthy animals. However, in the DM-model animals, the postprandial hypoglycemic effect of erythritol and acarbose became significant (Figure 1C). A significant decline in AUC was observed for both erythritol and acarbose-treated groups, demonstrating both drugs to exert an in vivo postprandial hypoglycemic effect (Figure 1D). This finding was contrary to a previous report that found erythritol treatment to have no effect on blood glucose ${ }^{20}$. However, in that study fasting blood glucose levels and not postprandial blood glucose were measured. In a very recent report a postprandial hypoglycemic effect of erythritol was shown in vivo ${ }^{21}$. However, this study focused specifically on the effects of erythritol on insulin resistance.

\section{Inhibition of $\alpha$-glucosidase activity by erythritol}

The ability of erythritol to inhibit $\alpha$-glucosidase was assessed. As shown in Figure 2, erythritol exhibited a strong inhibitory effect displaying an $\mathrm{IC}_{50}$ value of 6.43 
$\mathrm{mg} / \mathrm{mL}(52.7 \mathrm{mM})$. This suggests that the mechanism by which erythritol exerts its postprandial hypoglycemic effect is potentially through direct inhibition of $\alpha$-glucosidase. In healthy animals, under the hypoglycemic effect of insulin, it is likely that blood glucose concentrations are controlled so well that erythritol is unable to influence postprandial blood glucose (Figure 1A and 1B). However in the DM-model mice, in the absence of sufficient glycemic control by insulin, a reduction in the production of intestinal glucose by erythritol-mediated $\alpha$-glucosidase inhibition could explain the significant difference observed.

To determine the mechanism of inhibition kinetic studies were performed. For these, erythritol was added at three different concentrations (i.e. 5, 10 and $15 \mathrm{mg} / \mathrm{mL}$ ) and the reacations performed a at five different pNPG (substrate) concentrations (i.e. 0.11-0.51 mM). Product formation was first plotted against time to obtain initial velocities, which were calculated by taking the linear part of the increasing absorbance. The reciprocal velocities were subsequently plotted against the reciprocal of substrate concentration to construct Lineweaver-Burk plots. The Lineweaver-Burk plots for $\alpha$-glucosidase inhibition by erythritol generated linear data with different granients, which intersected at the same point on $\mathrm{Y}$-axis (indicative of an increased $\mathrm{K}_{\mathrm{m}}$ and unchanged $\mathrm{V}_{\max }$ ), indicating competitive inhibition. The Ki value was calculated to be $38.98 \mathrm{mM}$ at $5 \mathrm{mg} / \mathrm{ml}$ (Figure 3).

\section{Computational modeling of the $\alpha$-glucosidase-erythritol complex}

A Ramachandran Plot, often used as a first check to verify predicted torsion 
angles in proteins, showed low energy conformations for $\varphi$ (phi) and $\psi$ (psi), which were used to represent the torsion angles on either side of alpha carbons in peptides. The Ramachandran Plot provided the local backbone conformation of each residue by graphical expression form. The $\varphi$ and $\psi$ torsion angles of a residue were represented as the points on the Ramachandran Plot, which also included a representation of the favorable and unfavorable regions for residues to aid determination of whether individual residues were built correctly. As shown in Figure 4, most of the residues resided in either the core or allowed region with the exception of 9 residues (marked as red triangles), which overall indicated that the quality of the docking model was very good.

Next, we carried out a Libdock protocol to study the interactions between the $\alpha$-glucosidase enzyme and its inhibitor erythritol. During catalysis the Glu277 residue of the enzyme forms a hydrogen bond with $\mathrm{O} 1$ of the glucose saccharide, with a bond distance of $2.8 \AA$. The lengths of hydrogen bonds between Asp352 OD1 and O3 of the glucose residue and between OD2 and O2 are 2.7 and $2.5 \AA$, respectively. Asp69 OD2 and Arg442 NH1 form hydrogen bonds with O4 of the glucose residue. Glu277, Asp352, Asp69 and Arg442 together form the active site of $\alpha$-glucosidase. For erythritol (shown in Figure 5), a hydrogen-bond network was found at the two ends of the molecule. The hydroxyl group on the $\mathrm{C} 1$ carbon atom connected to Asp69 and Arg446 by hydrogen-bonds through the H113 water molecule. Another hydroxyl group on the C4 carbon atom made similar connections with Asp215, Arg213, Asp352 through H132 water molecules. It was clearly observed in our model that the active 
241 site of the enzyme was occupied by erythritol. We speculated that these H-bonds

242 provide the main electrostatic means to aid erythritol binding at the active pocket and

243 facilitate its demonstrated inhibitory activity.

244 In order to gain more information as to the chemical properties of the

245 erythritol-enzyme interactions, the binding free energy was decomposed into

246 inhibitor-residue pairs. The resultant quantitative data (shown in Table 1) was very

247 useful for understanding how erythritol could bind in the active pocket of $248 \alpha$-glucosidase. It was demonstrated that the major binding energy was electrical free

249 energy and the majority dissociation energy was polar solvation free energy. The van

250 der Waals free energy, electrical free energy and non-polar solvation free energy were

251 sufficient to overcome the polar solvation free energy to promote erythritol binding.

252 To deepen our understanding on forces stabilizing the binding of erythritol to the

253 enzyme, we decomposited the free energy of the residues in the active pocket

254 proposed to interact with erythritol (Figure 6). It was demonstrated that Asp 215,

255 Asp69 and Arg446 residues were likely to contribute the largest total binding free

256 energy. By decomposition, we found that these three residues provided a high

257 proportion of electrical free energy to promote erythritol binding in the active pocket,

258 which we propose is provided by hydrogen-bonds. Moreover, these three residues

259 undertake the largest polar solvent decomposition free energy. For Asp 352, Val 109

260 and Arg213 residues, the most important binding energy was solar solvent free energy,

261 while the main decomposition free energy was electrical free energy. In addition, we

262 found that all the four kinds of energy could promote inhibitor-binding by His351 and 
Val216 residues, in which van der Waals and polar solvent free energy provided the most energy.

In summary, we have shown using an alloxan-induced DM mouse model that erythritol treatment significantly reduces postprandial blood glucose at the dose of $500 \mathrm{mg} / \mathrm{Kg}$ compared with acarbose $(4 \mathrm{mg} / \mathrm{Kg})$, but has no significant effects on healthy animals. Biochemical analysis indicated that erythritol exerts its hypoglycemic effect by inhibiting $\alpha$-glucosidase in the competitive manner. Molecular docking of erythritol to the $\alpha$-glucosidase, isomaltase from $S$. cerevisiae revealed that the $\mathrm{C} 1$ hydroxyl and $\mathrm{C} 4$ hydroxyl groups of erythritol may form hydrogen-bonds with Asp69, Arg446, Asp215, Arg213 and Asp352 residues though H113 and H132 water molecules to competitively occupy the active pocket. An energy decomposition study indicated that electrical free energy provided the majority of the binding free energy and the polar solvent free energy provided the majority dissociation energy. The Asp 215, Asp69 and Arg446 residues contributed the strongest total binding free energy. It is hoped that these findings will stimulate further work into the role of erythritol in the management of DM and its potential use as a therapeutic food additive.

\section{Acknowledgments}

This work was supported by grants from Natural Science Foundation of Qinghai (No. 2016-ZJ-942Q), West Light Foundation of the Chinese Academy of Sciences (No. Y629071211), National Natural Science Foundation of China (No. 31701243), International Cooperative Projects of Qinghai province (No. 2017-HZ-811), Project of 
285 Discovery, Evaluation and Transformation of Active Natural Compounds, Strategic

286 Biological Resources Service Network Program of Chinese Academy of Sciences (No. 287 ZSTH-027), Major Special Science and Technology Projects in Qinghai Province 288 (2014-GX-A3A-01).

$290 \quad$ Figure captions

291 Figure 1 (A) After intragastric administration of erythritol $(500 \mathrm{mg} / \mathrm{Kg})$ and acarbose $292(4 \mathrm{mg} / \mathrm{Kg})$ to healthy mice, the blood glucose was monitored at different time points.

293 (B and D) the area under concentration-time curve (AUC) were calculated to evaluate 294 the postprandial blood glucose changes. (C) After intragastric administration of 295 erythritol $(500 \mathrm{mg} / \mathrm{Kg})$ and acarbose $(4 \mathrm{mg} / \mathrm{Kg})$ to diabetes mellitus mice, the blood 296 glucose was monitored at different time points.

Figure 2. Inhibition of $\alpha$-glucosidase by acarbose (A) and erythritol (B). Data was 298 fitted using a logistic function to calculate the $\mathrm{IC}_{50}$ values. The detailed inhibition data 299 is presented.

Figure 3. Lineweaver-Burk plot of erythritol-mediated $\alpha$-glucosidase inhibition. Data is plotted as a double reciprocal of initial reactions velocities versus concentration of the substrate, pNPG.

Figure 4. Ramachandran contours were used to test the quality of the presented molecular model. Green dots indicate the position of residues in the core region, while red triangles highlight the outlying residues.

306 Figure 5. Molecular model of erythritol-binding at the catalytic active pocket of 
307

308

309

310

311

312

313

314 Table 1 Total binding free energy decomposition $(\mathrm{kJ} / \mathrm{mol})$

$\alpha$-glucosidase. The interacting protein residues, Asp 215, Asp69, Arg446, Asp 352, Val 109, Arg213, His351 and Val216 are highlighted.

Figure 6. Decomposition of the calculated free energies of the Asp 215, Asp69, Arg446, Asp 352, Val 109, Arg213, His351 and Val216 residues, which are proposed to contribute to the binding of erythritol to $\alpha$-glucosidase.

\begin{tabular}{ccc}
\hline Energy Component & Average & Std. Dev. \\
\hline VDWAALS & -9.8652 & 3.3202 \\
EEL & -69.2587 & 6.6086 \\
EGB & 58.9938 & 4.1258 \\
ESURF & -3.2684 & 0.1329 \\
DELTA G gas & -79.1239 & 5.4402 \\
DELTA G solv & 55.7253 & 4.1494 \\
DELTA TOTAL & -23.3986 & 4.9532 \\
\hline
\end{tabular}

VDWAALS is total van der waals free energy. EEL is total electrical free energy.

316 EGB is total polar solvation energy. ESURF is total non-polar solvation energy. 
References

1. Avogaro, A.; Fadini, G. P., The effects of dipeptidyl peptidase-4 inhibition on microvascular diabetes complications. Diabetes care 2014, 37 (10), 2884-94.

2. Pippitt, K.; Li, M.; Gurgle, H. E., Diabetes Mellitus: Screening and Diagnosis. American family physician 2016, 93 (2), 103-9.

3. Vasiliadis, I.; Kolovou, G.; Mavrogeni, S.; Nair, D. R.; Mikhailidis, D. P., Sudden cardiac death and diabetes mellitus. Journal of diabetes and its complications 2014, $335 \quad 28(4), 573-9$.

4. Walker, A. M.; Cubbon, R. M., Sudden cardiac death in patients with diabetes mellitus and chronic heart failure. Diabetes \& vascular disease research 2015, 12 (4), 228-33.

5. Biadgo, B.; Abebe, M., Type 2 Diabetes Mellitus and Its Association with the Risk of Pancreatic Carcinogenesis: A Review. The Korean journal of gastroenterology

$341=$ Taehan Sohwagi Hakhoe chi 2016, 67 (4), 168-77.

342 6. Islam, M. S., Fasting blood glucose and diagnosis of type 2 diabetes. Diabetes 
research and clinical practice 2011, 91 (1), e26.

7. DeFronzo, R. A.; Abdul-Ghani, M., Assessment and treatment of cardiovascular risk in prediabetes: impaired glucose tolerance and impaired fasting glucose. The American journal of cardiology 2011, 108 (3 Suppl), 3b-24b.

8. Huang, Y.; Cai, X.; Mai, W.; Li, M.; Hu, Y., Association between prediabetes and risk of cardiovascular disease and all cause mortality: systematic review and meta-analysis. BMJ (Clinical research ed.) 2016, 355, i5953.

9. Gerich, J. E., Postprandial hyperglycemia and cardiovascular disease. Endocrine practice : official journal of the American College of Endocrinology and the American Association of Clinical Endocrinologists 2006, 12 Suppl 1, 47-51.

10. Mah, E.; Bruno, R. S., Postprandial hyperglycemia on vascular endothelial function: mechanisms and consequences. Nutrition research (New York, N.Y.) 2012, $32(10), 727-40$.

11. Fiorentino, T. V.; Prioletta, A.; Zuo, P.; Folli, F., Hyperglycemia-induced oxidative stress and its role in diabetes mellitus related cardiovascular diseases. Current pharmaceutical design 2013, 19 (32), 5695-703.

12. Ceriello, A.; Genovese, S., Atherogenicity of postprandial hyperglycemia and lipotoxicity. Reviews in endocrine \& metabolic disorders 2016, 17 (1), 111-6.

13. Hanefeld, M.; Temelkova-Kurktschiev, T., Control of post-prandial hyperglycemia--an essential part of good diabetes treatment and prevention of cardiovascular complications. Nutrition, metabolism, and cardiovascular diseases : NMCD 2002, 12 (2), 98-107. 
14. Park, M. H.; Ju, J. W.; Park, M. J.; Han, J. S., Daidzein inhibits carbohydrate digestive enzymes in vitro and alleviates postprandial hyperglycemia in diabetic mice. European journal of pharmacology 2013, 712 (1-3), 48-52.

15. Kalra, S.; Chadha, M.; Sharma, S. K.; Unnikrishnan, A. G., Untapped diamonds for untamed diabetes: The alpha-glucosidase inhibitors. Indian journal of endocrinology and metabolism 2014, 18 (2), 138-41.

16. Dewinter, L.; Casteels, K.; Corthouts, K.; Van de Kerckhove, K.; Van der Vaerent, K.; Vanmeerbeeck, K.; Matthys, C., Dietary intake of non-nutritive sweeteners in type 1 diabetes mellitus children. Food additives \& contaminants. Part A, Chemistry, analysis, control, exposure \& risk assessment 2016, 33 (1), 19-26.

17. Munro, I. C.; Berndt, W. O.; Borzelleca, J. F.; Flamm, G.; Lynch, B. S.; Kennepohl, E.; Bar, E. A.; Modderman, J., Erythritol: an interpretive summary of biochemical, metabolic, toxicological and clinical data. Food and chemical toxicology : an international journal published for the British Industrial Biological Research Association 1998, 36 (12), 1139-74.

18. Yokozawa, T.; Kim, H. Y.; Cho, E. J., Erythritol attenuates the diabetic oxidative stress through modulating glucose metabolism and lipid peroxidation in streptozotocin-induced diabetic rats. Journal of agricultural and food chemistry $\mathbf{2 0 0 2 ,}$ $50(19), 5485-9$.

19. Zeng, L.; Zhang, G.; Lin, S.; Gong, D., Inhibitory Mechanism of Apigenin on alpha-Glucosidase and Synergy Analysis of Flavonoids. Journal of agricultural and food chemistry 2016, 64 (37), 6939-49. 
387 20. Brusati, V.; Jozwik, M.; Jozwik, M.; Teng, C.; Paolini, C.; Marconi, A. M.;

388 Battaglia, F. C., Fetal and maternal non-glucose carbohydrates and polyols 389 concentrations in normal human pregnancies at term. Pediatric research 2005, 58 (4), $390 \quad 700-4$.

391 21. Chukwuma, C. I.; Mopuri, R.; Nagiah, S.; Chuturgoon, A. A.; Islam, M. S., 392 Erythritol reduces small intestinal glucose absorption, increases muscle glucose 393 uptake, improves glucose metabolic enzymes activities and increases expression of 394 Glut-4 and IRS-1 in type 2 diabetic rats. European journal of nutrition 2017. 


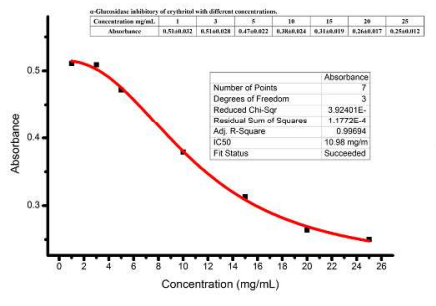

Inhibiting $\alpha$-glucosidase

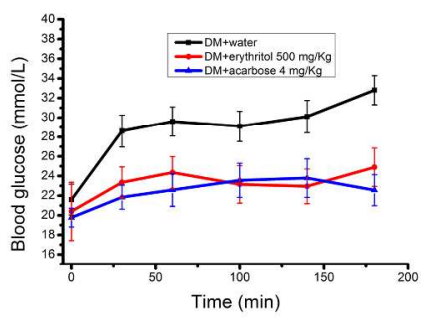

Reducing postprandial blood glucose

Graphic abstract

$244 \times 78 \mathrm{~mm}(300 \times 300 \mathrm{DPI})$ 

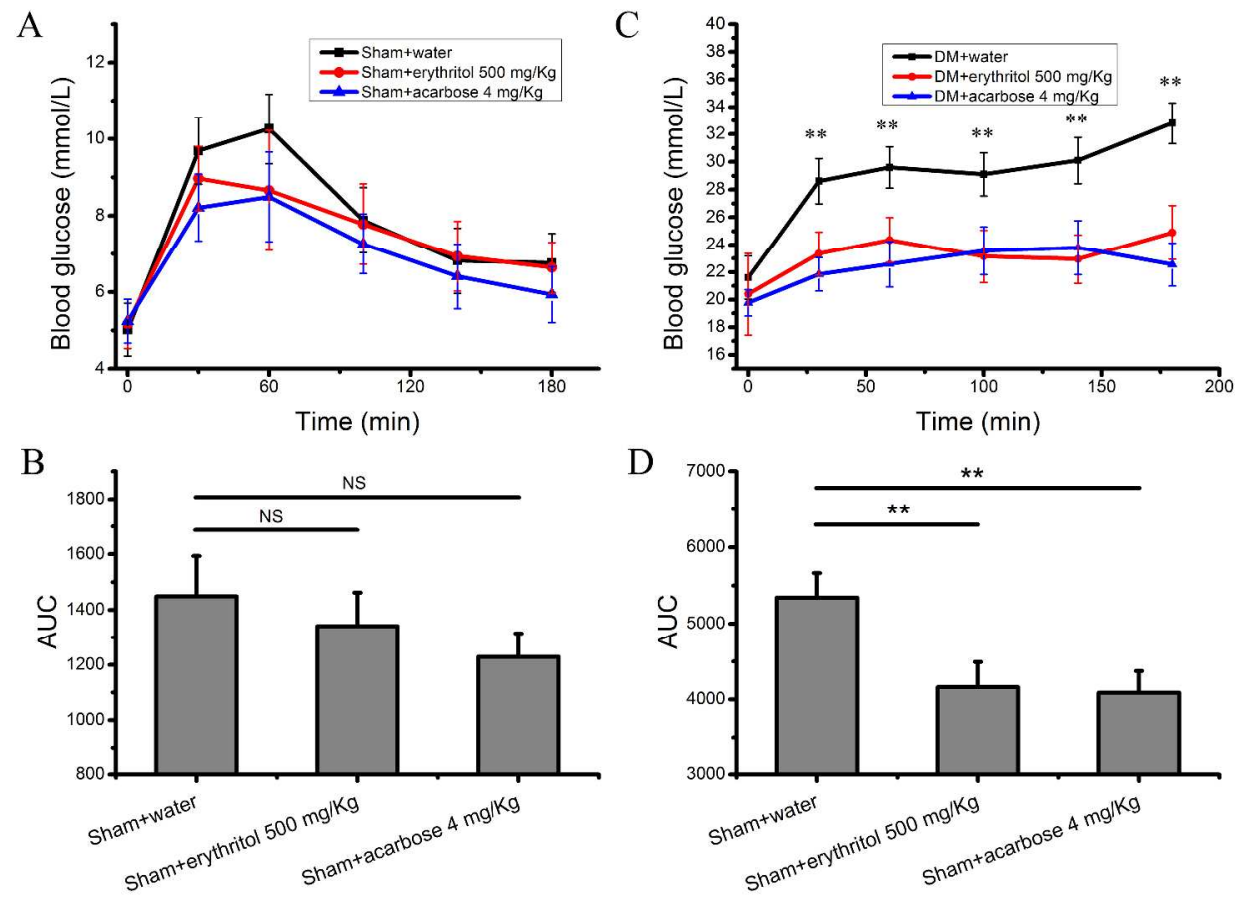

Figure 1 (A) After intragastric administration of erythritol $(500 \mathrm{mg} / \mathrm{Kg})$ and acarbose $(4 \mathrm{mg} / \mathrm{Kg})$ to healthy mice, the blood glucose was monitored at different time points. (C) After intragastric administration of erythritol $(500 \mathrm{mg} / \mathrm{Kg})$ and acarbose $(4 \mathrm{mg} / \mathrm{Kg})$ to diabetes mellitus mice, the blood glucose was monitored at different time points. (B and D) the area under concentration-time curve (AUC) were calculated to evaluate the postprandial blood glucose changes. 

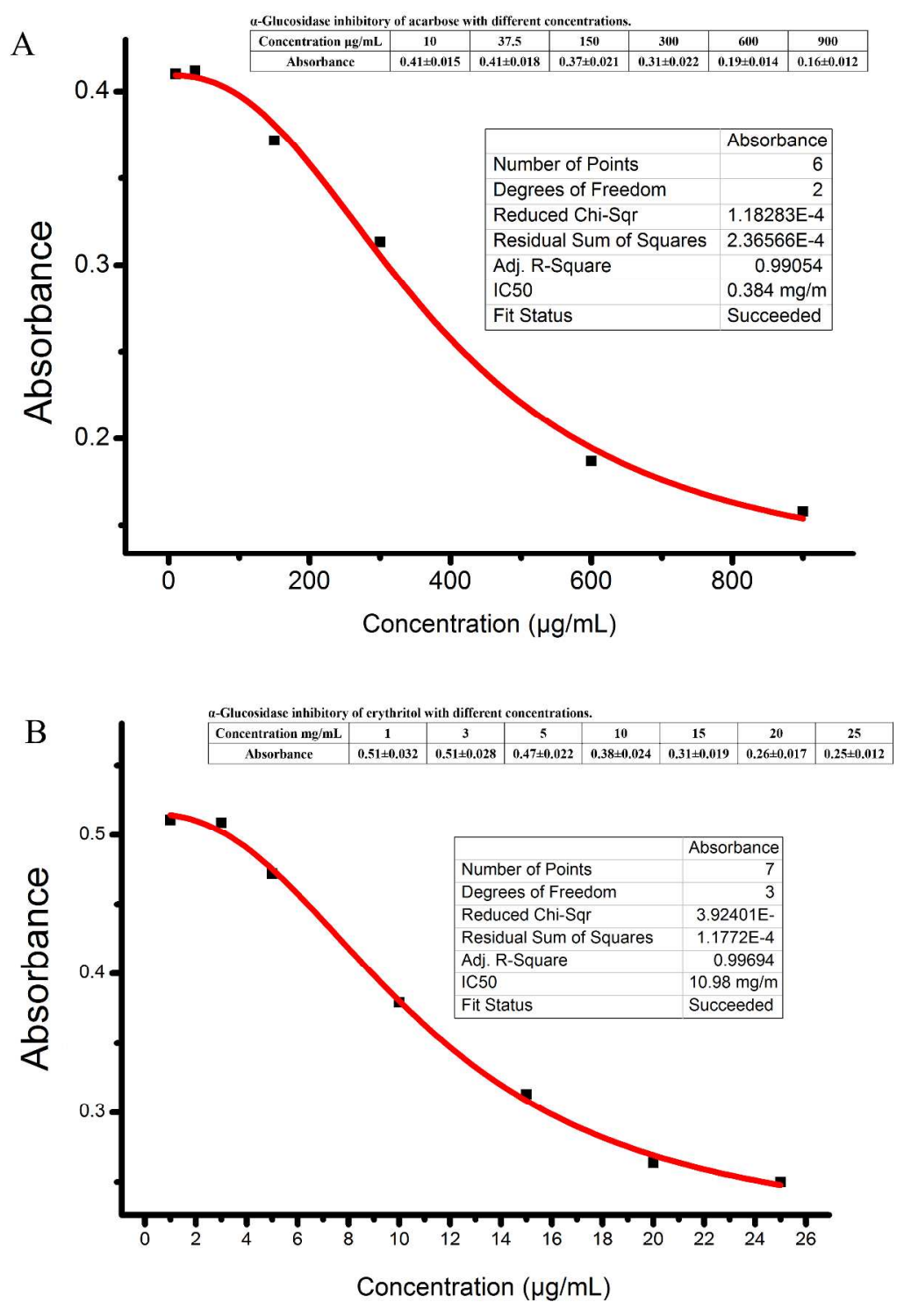

Figure 2 the inhibition data of acarbose (A) and erythritol (B) with different concentrations was fitted with logistic function to calculate the IC50 value. The detailed inhibition data was at the head of each figures.

$$
296 \times 419 \mathrm{~mm} \text { (300 x } 300 \text { DPI) }
$$




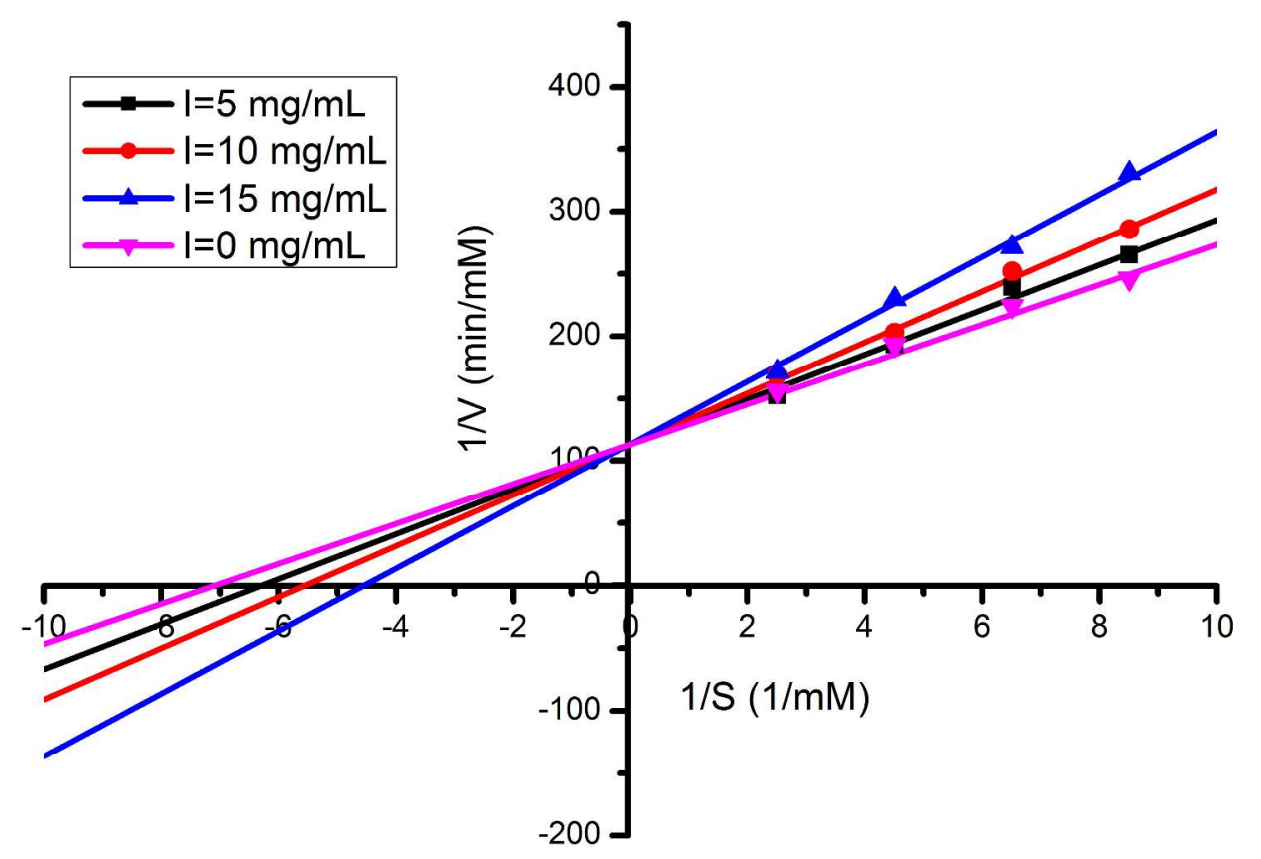

Figure 3 Lineweaver-Burk plot of erythritol against a-glucosidase at different concentrations of pNPG.

$288 \times 200 \mathrm{~mm}(300 \times 300 \mathrm{DPI})$ 


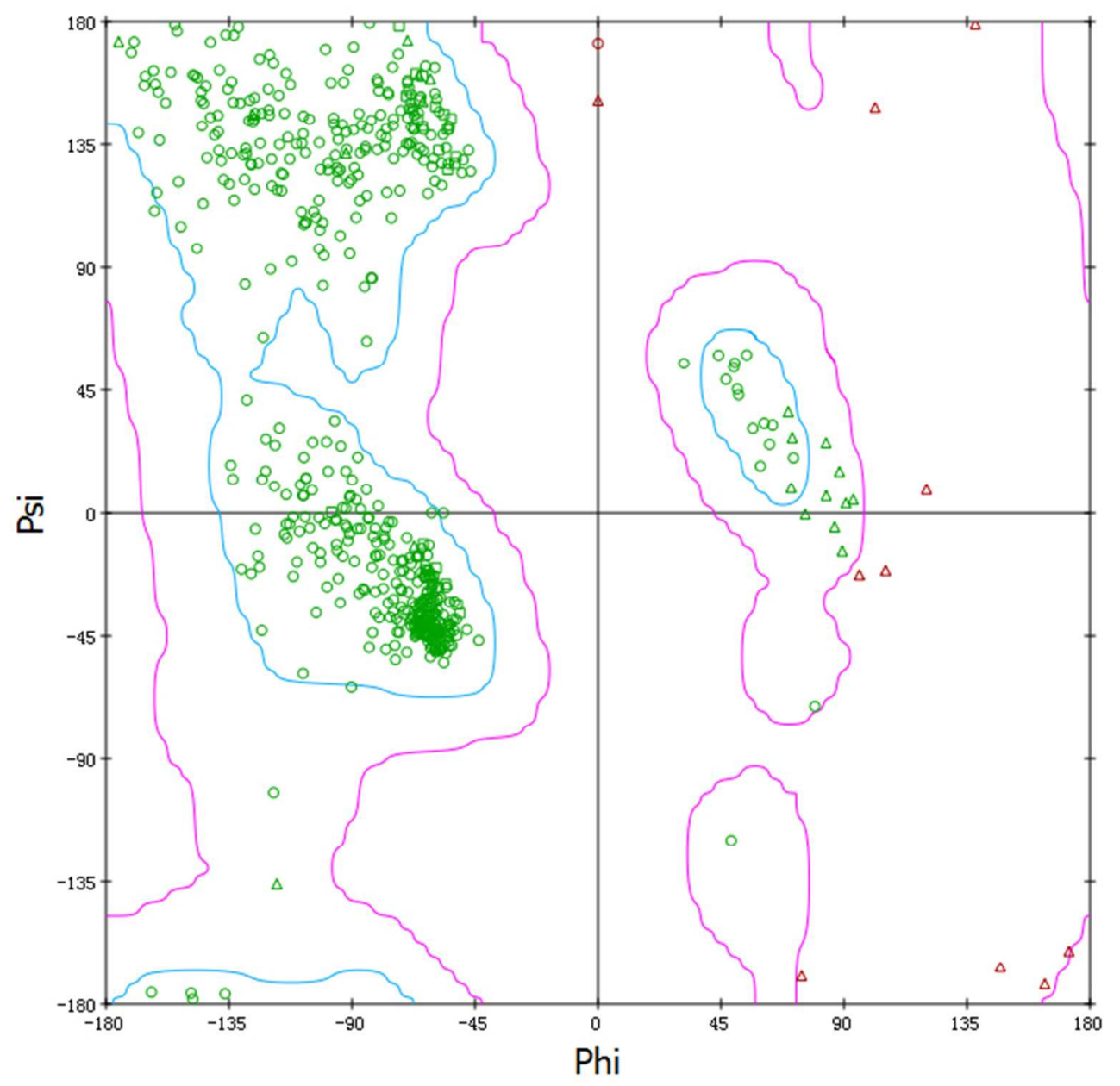

Figure 4 Ramachandran contours is used to test the quality of newly developed model. Green dots donate the position of residues in core region. While, red spots sign highlights the outliers.

$183 \times 176 \mathrm{~mm}(96 \times 96 \mathrm{DPI})$ 


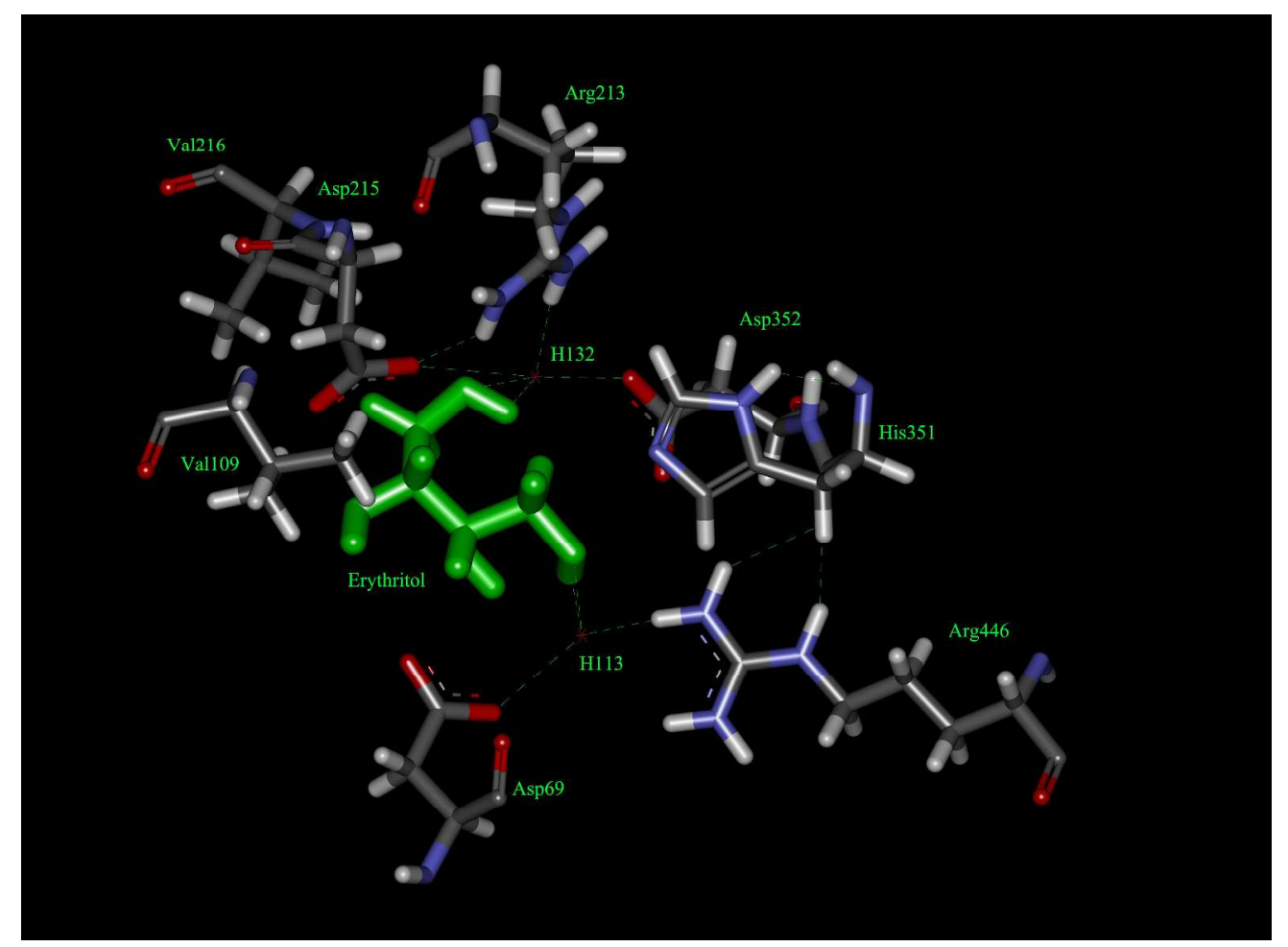

Figure 5 the interaction mode of erythritol in the catalytic active pocket of a-glucosidase with Asp 215, Asp69, Arg446, Asp 352, Val 109, Arg213, His351 and Val216.

$1587 \times 1174 \mathrm{~mm}(96 \times 96 \mathrm{DPI})$ 

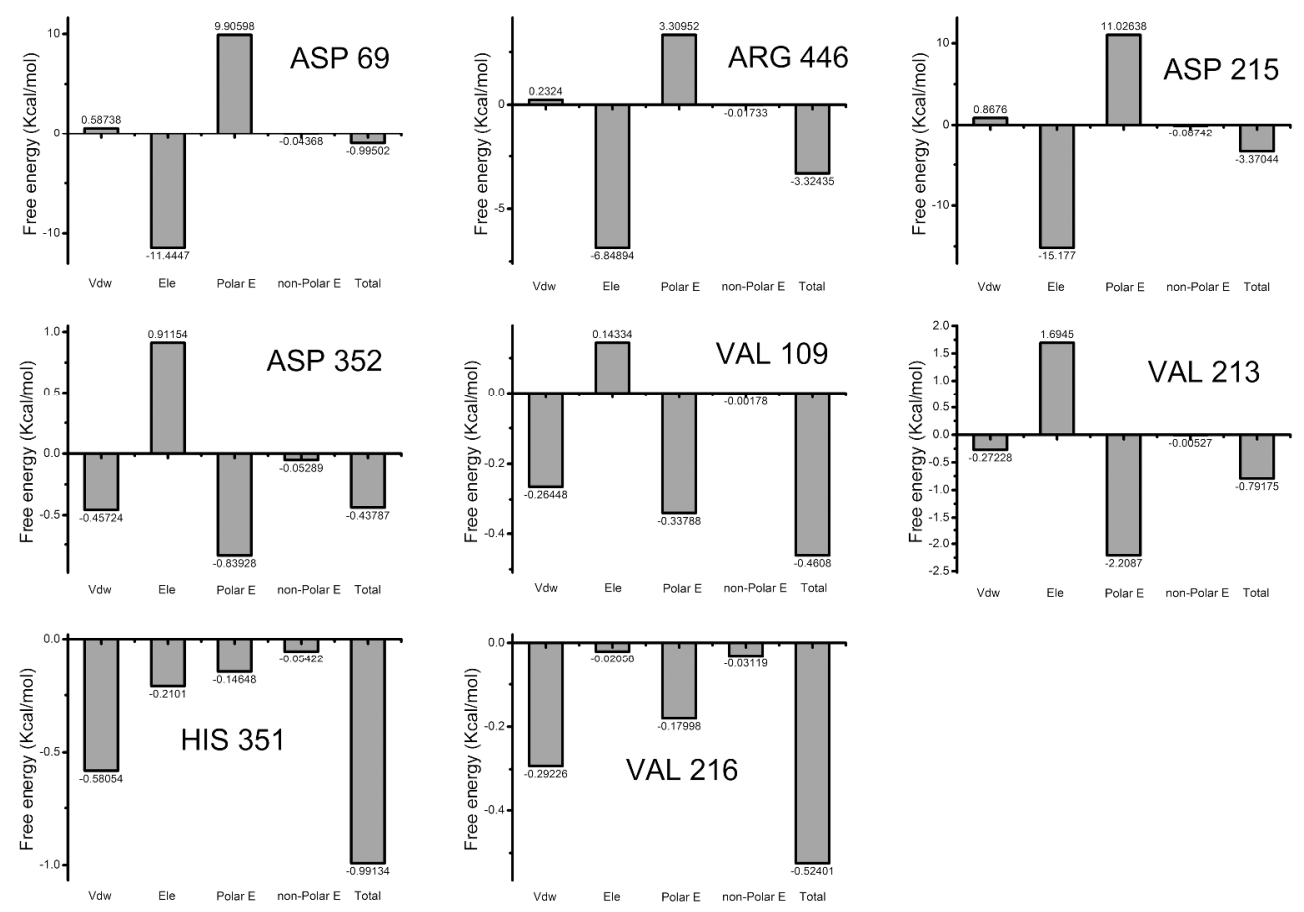

Figure 6 Decomposition of the free energy of Asp 215, Asp69, Arg446, Asp 352, Val 109, Arg213, His351 and Val216.

$423 \times 298 \mathrm{~mm}(300 \times 300 \mathrm{DPI})$ 